\title{
MONITORING CYANOBACTERIA BLOOM IN TAIHU LAKE BY HIGH-RESOLUTION GEOSTATIONARY SATELLITE GF4
}

\author{
Jian Liu \\ Surveying and Mapping Institute Lands and Resource Department of Guangdong Province, Guangzhou 510500, China \\ 11410856@qq.com
}

KEY WORDS: Cyanobacteria Bloom, Taihu Lake, GF4, Geosynchronous Earth Orbit, Remote Sensing

\begin{abstract}
:
The high-resolution remote-sensing satellite, GF4 PMS, of China's geosynchronous earth orbit was successfully launched on December 29, 2015. Its high spatial resolution and high temporal resolution allow GF4 PMS to play a very important role in water environment monitoring, especially in the dynamic monitoring of lake and reservoir cyanobacteria blooms. As GF4 PMS has just been launched, there is still relatively little related research, and the practical application effect of GF4 PMS in the extraction of cyanobacteria blooms remains to be further tested. Therefore, in this study, the method and effect of GF4 PMS application in cyanobacteria bloom monitoring were studied in Taihu. It turned that GF4 PMS can be applied to the dynamic monitoring of the distribution of cyanobacteria blooms in Taihu, thereby finding the temporal and spatial variation of the distribution of cyanobacteria blooms.
\end{abstract}

\section{INTRODUCTION}

The high-resolution remote-sensing satellite, GF4 PMS, of China's geosynchronous earth orbit was successfully launched on December 29, 2015 (Wang et al. 2017). The GF4 satellite has an infrared camera (InfraRed Scanner, IRS) and a panchromatic multispectral camera (Panchromatic and Multispectral Scanner, PMS). GF4 PMS has five bands: panchromatic band, blue band, green band, red band, and nearinfrared band. The ground spatial resolution of each of these bands is $50 \mathrm{~m}$, which is the highest spatial resolution in a remote-sensing satellite operated in the earth's synchronous orbit. Its high spatial resolution and high temporal resolution allow GF4 PMS to play a very important role in water environment monitoring, especially in the dynamic monitoring of lake and reservoir cyanobacteria blooms.

Cyanobacteria bloom is a phenomenon in which cyanobacteria proliferate and float on the surface of water. Cyanobacteria bloom is very harmful, especially as the bloom can easily produce algae toxin, which severely affects the water quality and safety of drinking water. Therefore, the dynamic monitoring of cyanobacteria blooms is of great significance to the prevention and control of cyanobacteria blooms.

Satellite remote sensing is an effective technique to dynamically monitor cyanobacteria bloom (Hu et al. 2010; Zhang and Duan 2008). Because the distribution of cyanobacteria blooms vary fast, it usually requires satellite remote sensing data with a high temporal resolution. For example, we can obtain MODIS two times per day and GOCI eight times per day. The resolution of MODIS for monitoring blooms is $250 \mathrm{~m}$ or $500 \mathrm{~m}$, and the resolution of GOCI is $500 \mathrm{~m}$. The spatial resolution is relatively low, and cannot be used for the extraction of small cyanobacteria blooms from large areas of water containing a relatively small bloom. Furthermore, as the extracted cyanobacteria bloom is often a mixture of land and cyanobacteria bloom, the actual distribution area of the cyanobacteria bloom may be misjudged.
GF4 PMS has a spatial resolution of $50 \mathrm{~m}$, and has the ability to obtain data several times per day. Thus, it is more advantageous than MODIS and GOCI in the dynamic monitoring of cyanobacteria blooms. As GF4 PMS has just been launched, there is still relatively little related research, and the practical application effect of GF4 PMS in the extraction of cyanobacteria blooms remains to be further tested. Therefore, in this study, the method and effect of GF4 PMS application in cyanobacteria bloom monitoring were studied in Taihu, which often occurs cyanobacteria bloom.

\section{DATA AND METHOD}

\subsection{Data}

We obtained 6 GF4 PMS images covering Taihu on four consecutive days of July 21-24, 2017, which were obtained at the following local times: 07-21 15:17, 07-22 09:11, 07-23 $09: 51,07-23$ 15:08, 07-24 09:29, and 07-24. The timecontinuous GF4 PMS image helps test the ability of the GF4 PMS image to monitor the dynamic distribution of cyanobacteria blooms.

\subsection{Image Preprocessing}

First, the GF4 PMS image should be geometric corrected. We first obtain the Landsat 8 OLI georeferenced image with near acquiring time, and resample it to $50 \mathrm{~m}$. Then, we made automatically registration of the GF4 PMS images and obtain geometric correction image.

The radiometric calibration of the GF4 PMS image is then carried out with the radiometric calibration coefficient provided by the China Resources Satellite Application Center.

Finally, atmospheric correction for the GF4 PMS image is carried out. Atmospheric correction in turbidity water often requires two short-wave infrared wavelengths, but GF4 PMS 
does not have these bands. Therefore, we performed a simplified Rayleigh scattering correction for GF4 PMS.

\subsection{Cyanobacteria Bloom Identification}

A cyanobacteria bloom is an algal floating on the surface of the water, and its spectral characteristics are similar to those of vegetation, that is, it has a near-infrared emission peak and red light as the reflection valley. In general, the near-infrared reflectance of the ordinary water body is lower than the redlight reflectance. Therefore, the use of the normalized vegetation index (NDVI) can distinguish between cyanobacteria bloom and other aquatic vegetation. As for the NDVI threshold bloom extraction, we try to compare several commonly used methods, including the fixed threshold method, gradient mode method, and visual interpretation method.

\section{RESULTS}

We compared three methods to determine the NDVI threshold: the fixed threshold method, gradient mode method, and visual interpretation method. The results showed that it is very difficult to obtain good bloom extraction using six images in the fixed threshold method; the gradient mode method cannot correctly extract the bloom in a small bloom range, and the visual interpretation method can best determine the threshold value, but requires a longer time. In this study, we need to accurately extract the distribution of the cyanobacteria bloom, so we ultimately choose the visual interpretation method.

We use visual interpretation to determine the threshold and to extract the cyanobacteria bloom map of the six images, which is shown in Figure 1. On the afternoon of July 21, the bloom is mainly distributed in the western Taihu coast and in the northwest of Zhushan Bay, and the bloom remains in this area until the morning of July 24. However, the area of the cyanobacteria blooms changed during this time. In the morning of July 22, the area of the cyanobacteria blooms increased significantly, and the area decreased from July 23 to July 24. During the afternoon of July 24, a new bloom area began to appear in the northern part of the lake.

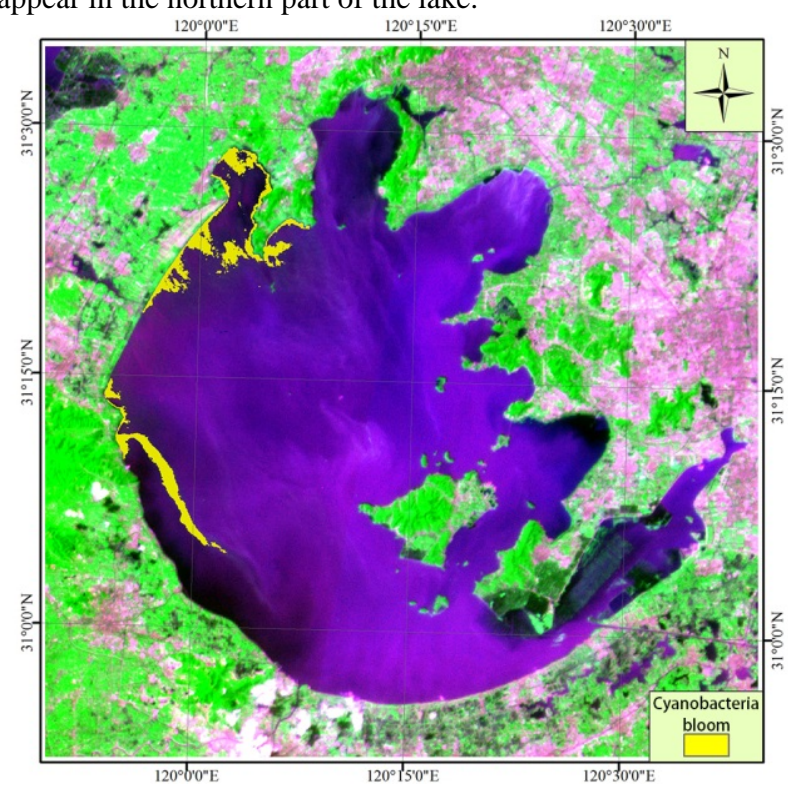

(a) $2016072115: 17$

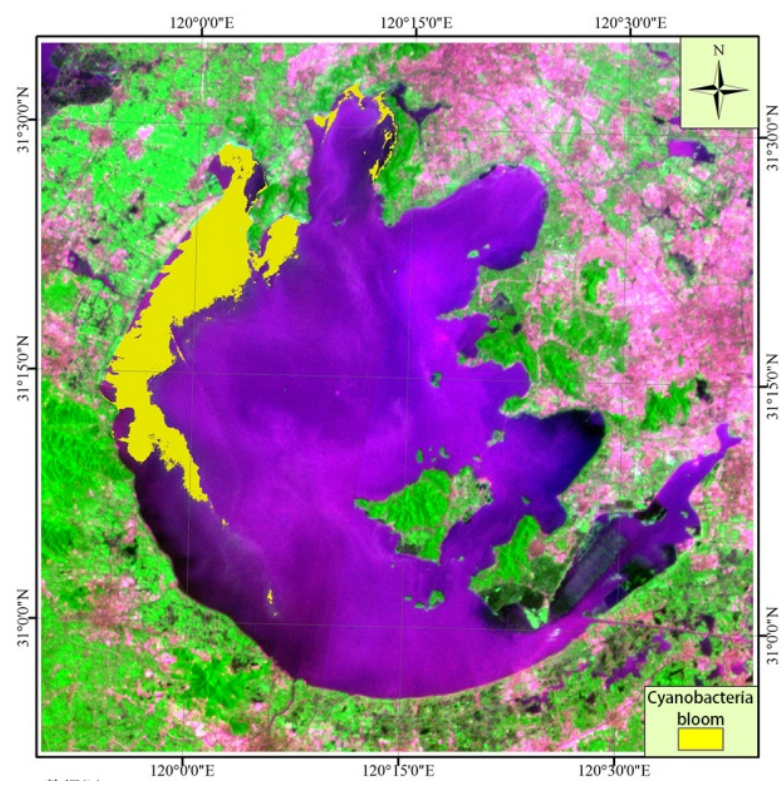

(b) 20160722 09:12

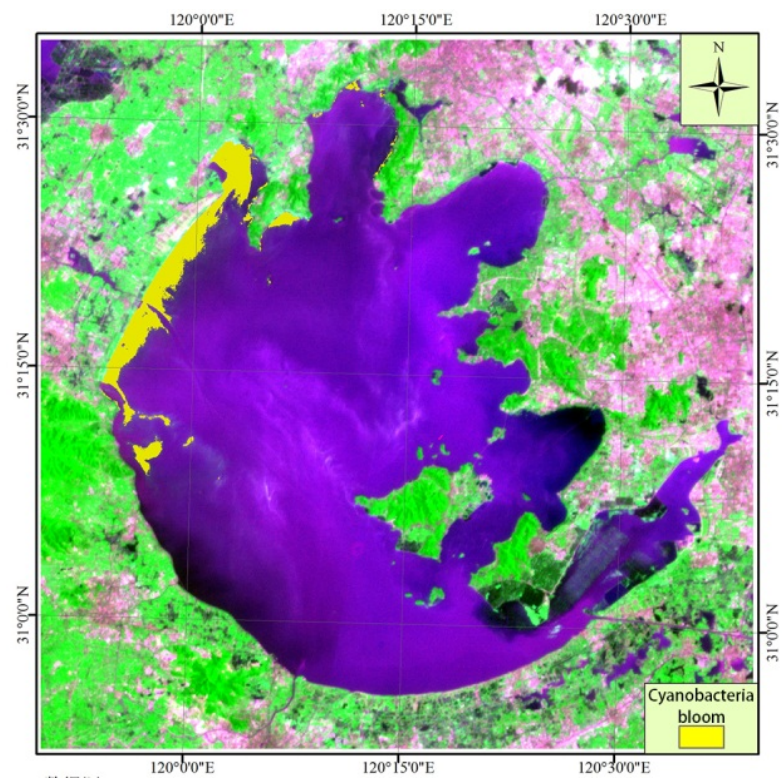

(c) 20160723 09:52 


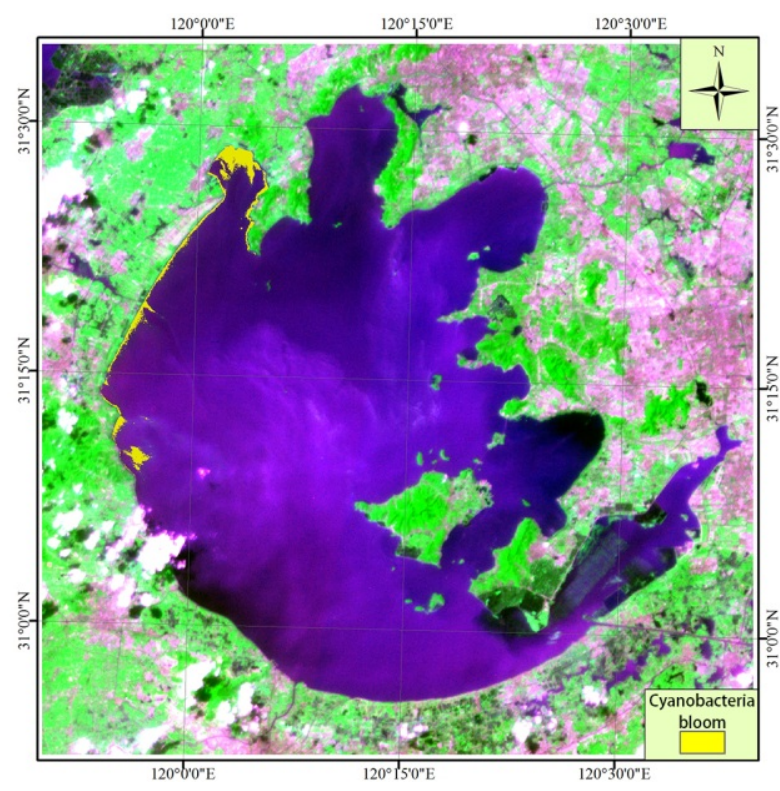

(d) 20160723 15:09

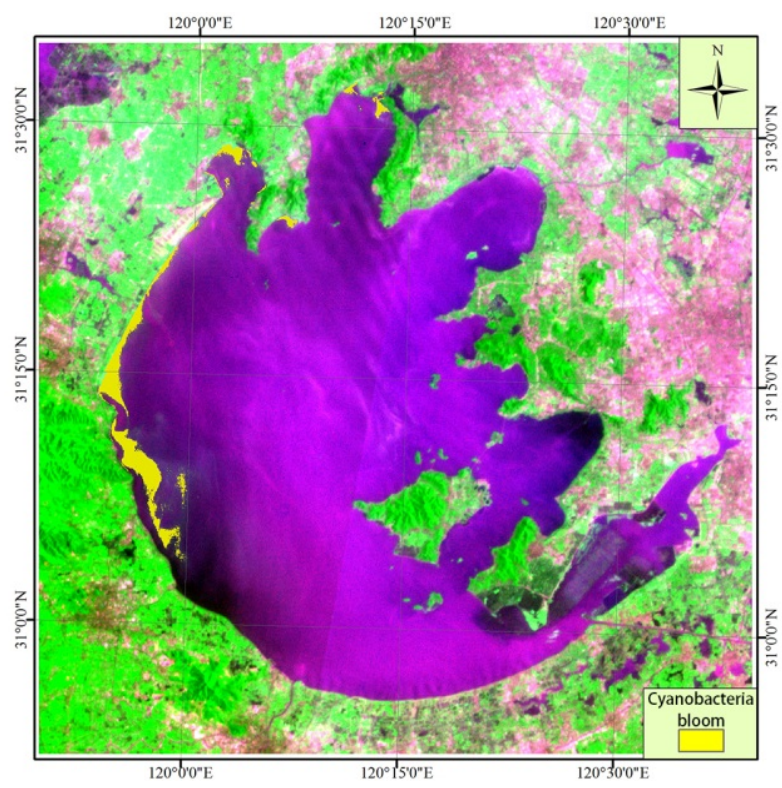

(e) 20160724 08:48

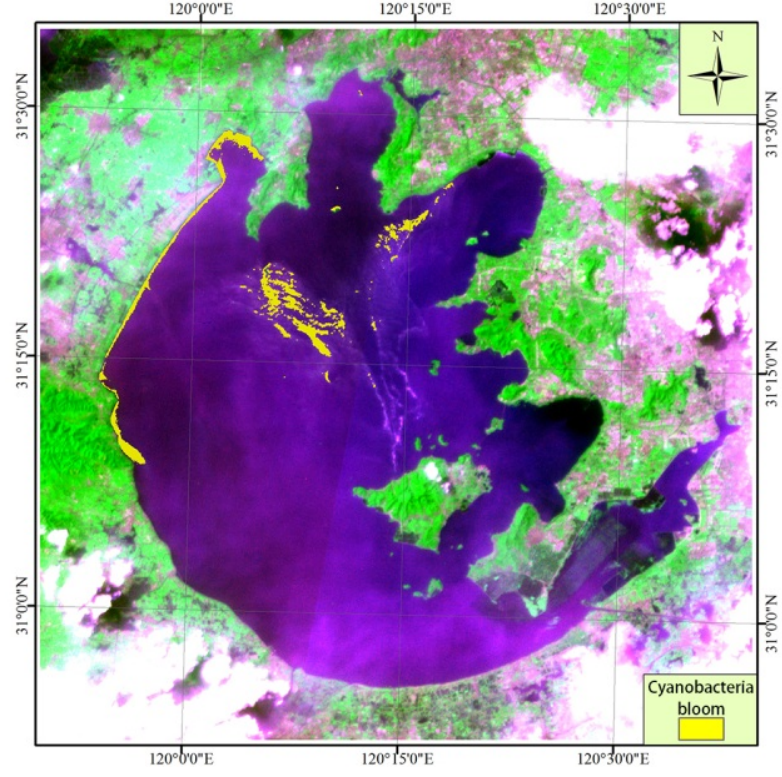

(f) 20160724 15:52

Figure 1. Cyanobacteria bloom identification results using GF4 images. The captions of each sub-figures are the image acquiring time in local time.

\section{CONCLUSIONS}

GF4 PMS has high spatial resolution and high time resolution, and has an important advantage in monitoring cyanobacteria blooms in the lake reservoir.

The NDVI threshold segmentation method can be applied to the extraction of cyanobacteria blooms in GF4 PMS data. The most reliable method for determining the NDVI threshold is visual interpretation.

GF4 PMS can be applied to the dynamic monitoring of the distribution of cyanobacteria blooms in Taihu, thereby finding the temporal and spatial variation of the distribution of cyanobacteria blooms.

\section{REFERENCES}

Hu Chuanmin, Lee Zhongping, Ma Ronghua, Yu Kun, Li Daqiu, Shang, Shaoling, 2010. Moderate resolution imaging spectroradiometer (MODIS) observations of cyanobacteria blooms in Taihu Lake, China. Journal of Geophysical Research, Oceans, 115(C4), pp. 303-306.

Wang Mi, Cheng Yufeng, Chang Xueli, Long Xiaoxiang, Li Qingpeng, 2017. High accuracy on-orbit geometric calibration of geostationary satellite GF4. Acta Geodaetica et Cartographica Sinica, 46(1), pp. 53-61.

Zhang Yuanzhi, Duan Hongtao, 2008. Cyanobacteria bloom detection and monitoring from satellite observations in the coastal region of Finland. Journal of Lake Sciences, 20(2), pp. 167-172. 